\title{
Scalp microbiome gets to the root of dandruff
}

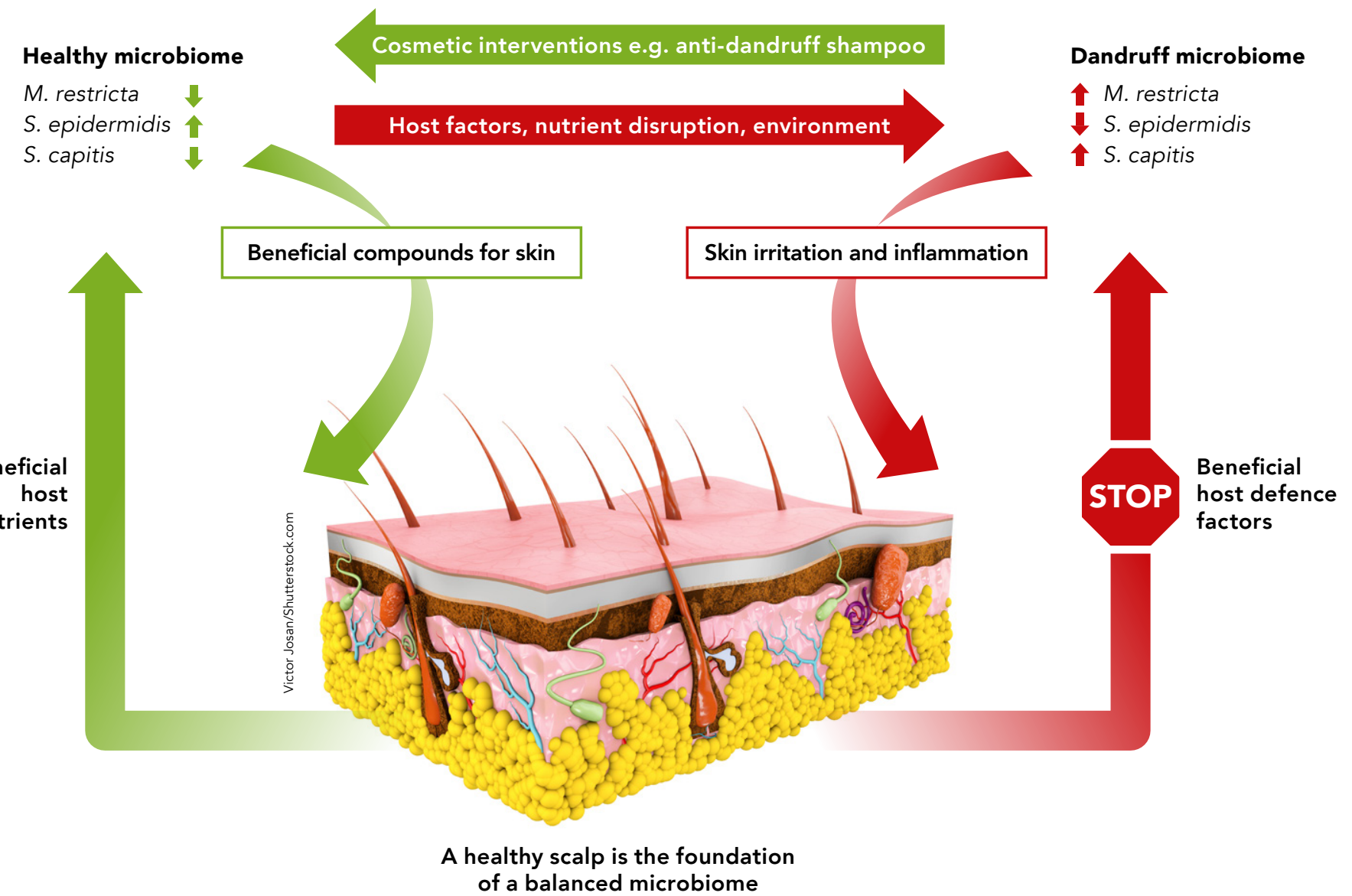

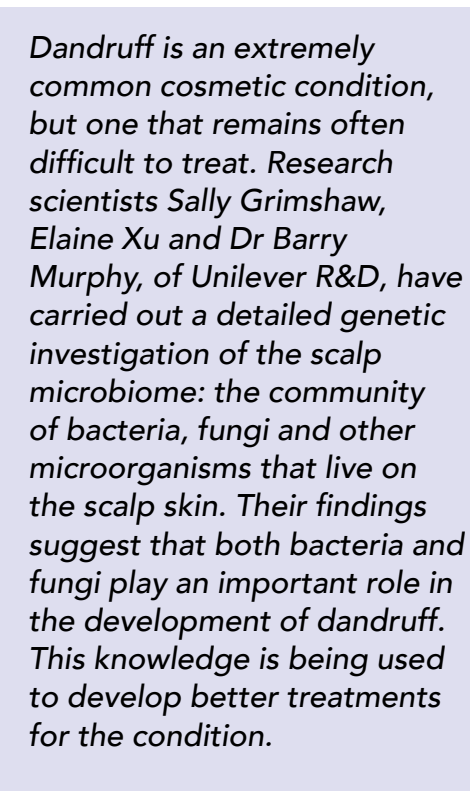

for the condition.

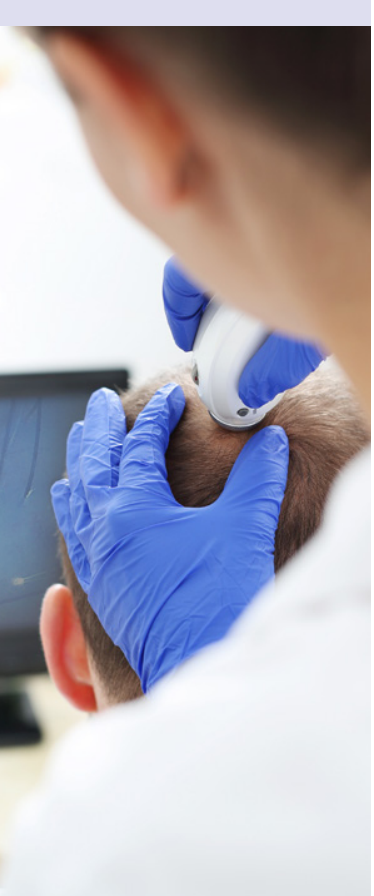

have all been linked to disturbances in the microbiome. It would probably Although no single defintivat list. for dandruff has been identified, researchers generally agree that certan changes or conditions are significant These include over-colonisation of the scalp by yeasts belonging to the genus Malassezia (yeasts are a type of fungus). Individuals may also be predisposed to dandruff due to changes in the activity of the sebaceous (oil) glands in the skin, and genetic factors that can result in a less robust skin barrier. Even the weather might play a role: many people find that their dandruff is worse in the winter months.

Fungi like Malassezia are normal residents of the skin's surface. Microorganisms like this are commensal, depend on their host, while causing no harm. However, wetimes the balance of the microbiome is disturbed, causing normally harmless microorganisms to become problematic. Multiple studies have found a link between the abundance of Malassezia and scalp health. When people with dandruff used an antifungal shampoo, the number of Malassezia on the scalp was reduced, and a decrease in skin flaking was seen - suggesting a relationship between the abundance of Malassezia and dandruff. However Malassezia is sometimes also present in high numbers on healthy scalps. This suggests that both a large Malassezia population and a predispo

\section{SEEKING THE CAUSES}

OF DANDRUFF

Recently, research interest has turned towards a possible role of bacteria in dandruff. This has been encouraged by one particular study which found that the link between bacteria and dandr might actually be stronger than the

Sally Grimshaw, Elaine Xu and Dr Barry Murphy, of Unilever R\&D, are investigating the scalp microbiome the genetic level, with a particular interest in bacteria. Recently, the team used a method called

next generation sequencing (NGS) to analyse the DNA of the complete scalp microbiome. simply put, NGS is a quick, relatively cheap DNA sequencing technique that can sequence "barcoding" marker genes present in bacteria and fungi, the sequencing of which faciltates their identifation Thanks to these
Neither bacteria nor fungi are solely responsible for dandruff; in fact, both are involved.

taken from two sites on the scalp of each person, one from the healthiest part of the scalp, and one from the least healthy. This allowed the microbiome to not only be compared between person. DNA was ext hame person. DNA was extracted from the The microbial DNA sequencence. then used to identify which species of bacteria and fungi resided on the scalps calles study participants. A technique callo quantative polymerase cha determine how was also used, to of each species were present

BACTERIA AND FUNGI: ARE THEY BOTH RESPONSIBLE? had predicted, the macrobs as they andruff differs from that althy scalp. Interestingly, it seem that neither bacteria nor fungi are solely responsible for dandruff; in fact, both are involved.

On both healthy and dandruff scalps, by ar the most abundant fungal resident is the yeast Malassezia restricta. For people without dandruff, M. restricta is found in similar quantities on both scalp. Howd levst heally parts of the scalp. However, on dandrutt scalps, the 


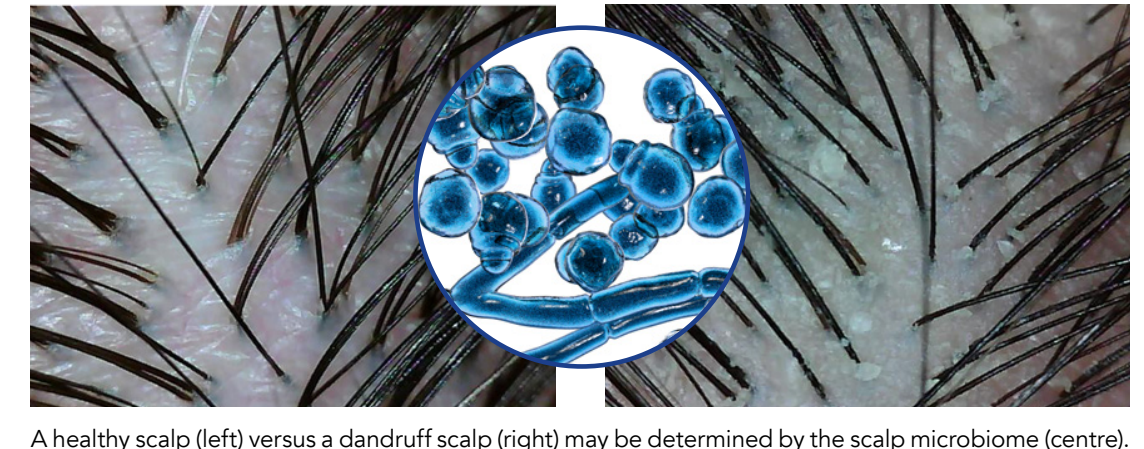

The number of bacteria differed both between healthy and dandruff scalps, and between different areas on the same scalp.

difference in abundance of $M$. restricta between the healhiest and least heal hy not only are some individuals me susceptible to dandruff than others, but that, for the same person, some parts of the scalp might be more prone to dandruff than others.

The team's work identified a further four known species of Malassezia in the scalp samples. They also uncovered anoth undefined species of Malassezia. Importantly, although this new yeast species was found in both groups of participants, it was far more abundant on the scalps of people with dandruff. Alone, this result is not enough to say dand hystios Ms wezia causes attention through future research.

So what about the bacteria? Staphylococcus and Cutibacterium proved to be the most abundant types of bacteria on both healthy and dandruff scalps. As with Malassezia, the number of staphylococci differed both between healthy and dandruff scalps, and between different areas on the same scalp. Significantly, this suggests that something local is happening to disrupt the microbiome, rather than a condition that affects the individual as a whole.

\section{A NEW APPROACH}

Grimshaw, Xu and Murphy's work is the first time that these combined approaches have been used to study
the scalp microbiome. The use of species of Staphylococcus, S. epidermid declines in number, while another eam suspect that the increases in both $M$. restricta and $S$. capitis seen in this study could be explained by both species having the ability to exploit changes in scalp biology. What these changes are damage to the skin surface, or changes in skin oil production, for example - could be revealed by further research.

The microbiome is a complex and sometimes delicate community of microorganisms. Successfully identifying the bacteria and fungi in the microbiomes of both healthy and dandruff scalps, as the team at Unilever have done, is the first step towards discovering why the microbiome can become unbalanced, dandruff, itch and dryness. With this drndrif, ich and dynss. Wh his products such as the Clear Advanced Protection anti-dandruff shampoo, Clear Scalp Clinic range and Living Proof Restore Dry Scalp Treatment that target the root cause of these conditions scalp microbiome.

$$
\begin{aligned}
& \text { might be linked to the development of target the root cause of these con } \\
& \text { dandruff. In a further result, the team and restore the equilibrium of the }
\end{aligned}
$$
found that, on a dandruff scalp, one

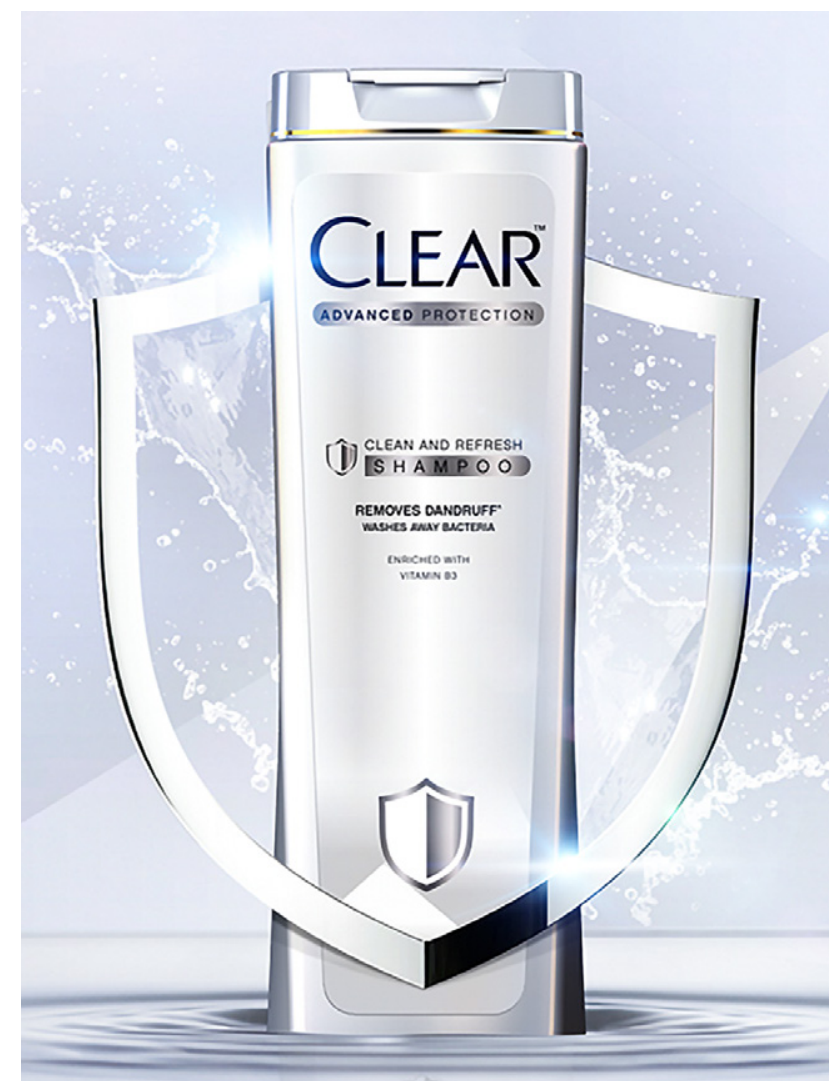

The Unilever team's work allows the development of targeted
anti-dandruff products such as those pictured here.

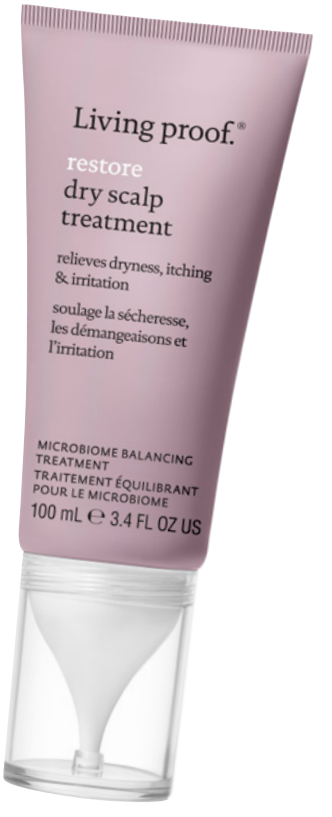

\section{Behind the Research}

(1)

Sally G

Grimshaw E: sally.grimshaw@unilever.com

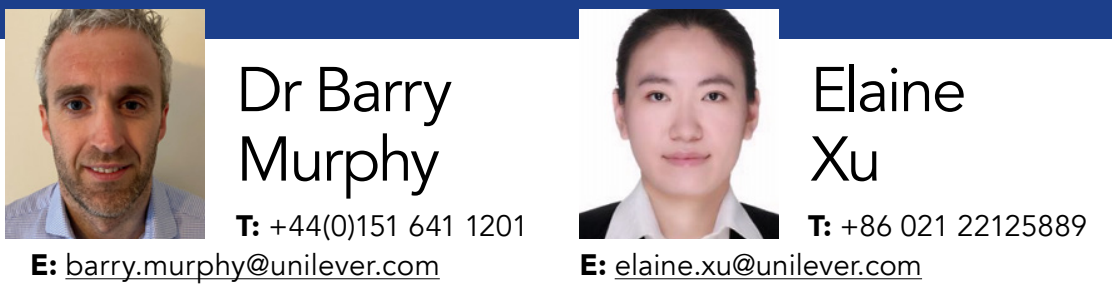

\section{Research Objectives}

The team at Unilever investigate the scalp microbiome to better understand the causes of dandruff.

\section{Detail}

Sally G. Grimshaw

Barry Murphy

Unilever R\&D Port Sunlight

Quarry Road East

Bebington

Wirral

H63 3JW

Elaine Xu

66, Linxin Rd.

China

Bio
Unilever has an established
R\&D global capability in Human
Microbiomics. In partnership with
leading experts in the field of Next
Generation Sequencing (NGS) and
Bioinformatics we have undertaken
numerous clinical studies to obtain a
deeper understanding of the role of
the microbiome in consumer relevant
conditions across our Beauty and
Personal Care division, examining the
microbiome associated with cosmetic
skin conditions, axillary malodour and
oral health.

More information www.clearhaircare.com

www.livingproof.com

Funding

The study was funded by

Collaborators

The University of Liverpool's Centre for (CGR),

Institute, Shenzhen, Guangdong

China; Eagle Genomics,

\section{References}

Grimshaw, SG, et al. 2019. The diversity and abundance

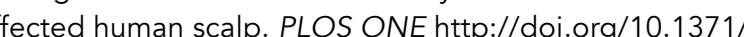
journal.pone.0225796

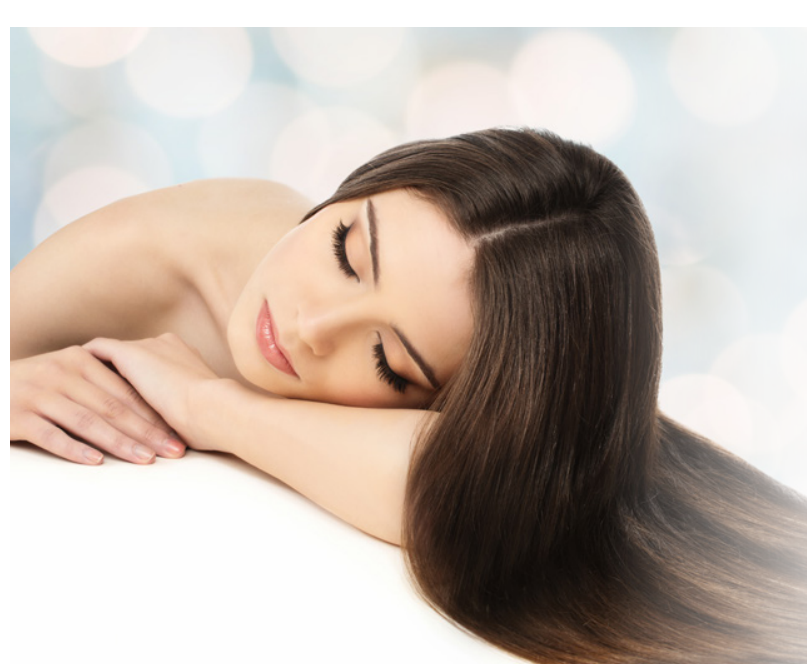

\section{Personal Response}

How might S. capitis interact with other aspects of the microbiome to cause dandruff?

II Interactions between different species of bacteria and fungi are complex and require further investigation to be fully understood. Micro-organisms consume provide the scalp with essential nutrients and also produce substances that can inhibit the growth of other microbes. However, the over or underproduction of these metabolites can have a detrimental impact on the skin of susceptible individuals. This balancing act is in the levels of $S$. capitis and $M$. restricta is an important indication of microbiome imbalance necessitating an intervention with anti-dandruff technology.

CLEAR 笅㩆

olary. Living proof. 